\title{
Pathogens, precipitation and produce prices
}

\author{
Crop production and food security remain one of the primary concerns in a changing world. Research and \\ comments in this issue highlight the various threats to our produce and the carry-over effects of food shocks.
}

W hile the direct and indirect impacts of the climate crisis on human health have long been documented, unsurprisingly, the last $\sim 18$ months have seen increased interest in the role of climate change in the spread of pathogens. Focus has been placed on the spread of insect (for example, ref. ${ }^{1}$ ) or mammalian vector populations ${ }^{2}$ under recent or future climate changes, but more bizarre stories have also been reported in the media. The concept of 'zombie-like pathogens' that could re-emerge from deep-cold slumbers was itself reawakened in the popular consciousness in 2016 when a 12-year-old boy died of anthrax (Bacillus anthracis). The presumed cause: a heatwave that thawed frozen Siberian soil, and with it, an infected reindeer carcass.

Often somewhat peripheral to these discussions lies the fact that pathogens are not only a detriment to human health through direct infection, but that they also exist as a major threat to global food supply. The Food and Agriculture Organization of the United Nations (FAO) estimates that plant diseases cost the global economy around US $\$ 220$ billion per year, with $20-40 \%$ of crop production lost to pests ${ }^{3}$. Unsurprisingly, there is already evidence that changing climate plays a role in the spread and management of plant pathogens and pests.

Wheat blast disease, caused by the fungus Magnaporthe oryzae Triticum, represents a severe threat to wheat production. Originally identified in Brazil, the disease was first reported in Bangladesh in 2016 and subsequently spread to neighbouring India, the world's second largest producer of wheat. Although the pathogen itself was likely transported into the region by anthropogenic activity, the 2016 outbreak was believed to be facilitated by an abnormally warm and humid pre-harvest season, linked to climate change ${ }^{4}$.

More broadly, a 2020 study in Nature Climate Change demonstrated that warming increases the abundance of soil-borne potential fungal plant pathogens ${ }^{5}$, while a 2013 study of 600 crop pests and pathogens ${ }^{6}$ showed that poleward movement of just under $3 \mathrm{~km}$ per year has been underway

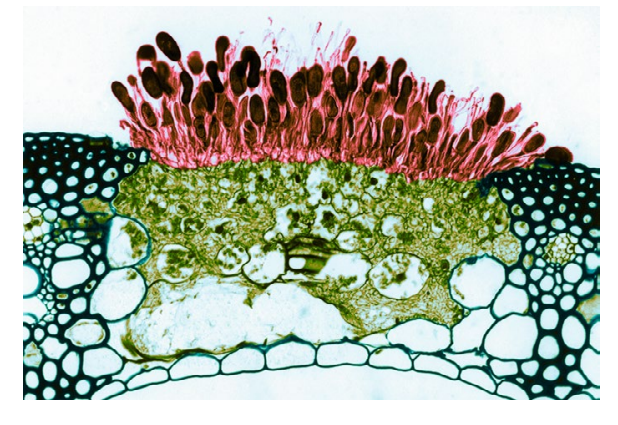

Black stem rust on a wheat leaf. Credit: Science history images / alamy stock photo

since 1960. In this month's issue, Chaloner and colleagues project the impact of future climates on both the productivity of 12 crop species and the temperature-dependent infection risk from 80 fungal and oomycete (fungus-like) plant pathogens. While the researchers find support for average global increases in yield for most crop species driven primarily by productivity boosts at high latitude - they also reveal expected increases in infection risks that will track these changes. See also the Saunders N\&Vs for further discussion.

Of additional concern is that these changes will also see marked shifts in the assemblages of pathogens at certain locations. In a similar vein, the 2013 work found differences in movement between broader taxonomic groups, and a recent study $^{7}$ showed that many plant-eating insect pest species have mixed responses (both increasing and decreasing) to change. Overall, these convoluted responses complicate costly management methods, which may be pest- or pathogen-specific.

Adding further to this complexity is the fact that plant pathogen changes will not be the only challenges facing crop production. Hotter, drier events, including prolonged droughts, will require further adaptation to ensure food security. Examples featured in this issue include a research article by Fischer and colleagues, which estimates that the probability of 'record-shattering' heat events - week-long heat extremes that break records by three or more standard deviations - will be up to 21 times more likely in the years 2051-2080 than during the last three decades. A Snapshot discusses some of the droughts that have impacted most continents throughout the first half of 2021. Southern Madagascar, for example, has experienced consecutive harvest failures and is in an extended and severe 'lean season' (the period between harvests), and this region will experience increased drought severity with warming. Importantly, droughts rarely occur in isolation, and the risk of simultaneous breadbasket failure has increased historically ${ }^{8}$.

Threats to food security represent a global issue, and, like many climate change impacts, have disproportionate effects on already disadvantaged groups. Beyond local impacts, agricultural disruptions caused by extreme weather events can have far-reaching carry-over economic repercussions, such as the 2010 droughts in Russia and eastern Europe that caused food shortages and price surges. Peersman and colleagues find that such disruptions cause economic impacts that are stronger for higher-income than lower-income countries, despite lower shares of food in household expenditures in the former.

The impacts of climate change on crop productivity and food security can be diverse and disastrous. While adaptation is needed in both the short and long term to mitigate the damage, it's clear that additional preventative strategies to buffer the shock of future change - such as the recently discussed diversification of food supply chains $^{9}$ - should also be planned.

Published online: 5 August 2021

https://doi.org/10.1038/s41558-021-01124-4

References

1. Iwamura, T., Guzman-Holst, A. \& Murray, K. A. Nat. Commun. 11, 2130 (2020).

2. Wang, Y. X. et al. Glob. Change Biol. (in the press).

3. New standards to curb the global spread of plant pests and diseases. FAO http://www.fao.org/news/story/en/item/1187738/ icode/ (2021).

4. Islam, M. T., Kim, K. H. \& Choi, J. Plant Pathol. J. https://doi. org/10.5423/PPJ.RW.08.2018.0168 (2019).

5. Delgado-Baquerizo, M. et al. Nat. Clim. Change 10, 550-554 (2020).

6. Bebber, D., Ramotowski, M. \& Gurr, S. Nat. Clim. Change 3, 985-988 (2013).

7. Lehmann, P. et al. Front. Ecol. Environ. 18, 141-150 (2020).

8. Gaupp, F. et al. Nat. Clim. Change 10, 54-57 (2020).

9. Gomez, M. et al. Nature 595, 250-254 (2021). 